\title{
Fissuration à chaud : des alliages organiques aux superalliages
}

\author{
Jean-Marie Drezet $^{1,2, a}$, Pierre-Daniel Grasso ${ }^{1}$ et Michel Rappaz ${ }^{1}$ \\ 1 Laboratoire de Métallurgie Physique, EPF-Lausanne, 1015 Lausanne, Suisse \\ 2 Calcom SA, Parc Scientifique, EPF-Lausanne, 1015 Lausanne, Suisse
}

Reçu le 10 juin 2003, accepté le 7 novembre 2003

\begin{abstract}
Résumé - L'apparition de fissures ou criques à chaud pendant la coulée des matériaux métalliques représente un défaut sérieux. L'EPF-Lausanne a développé un critère d'initiation de fissures qui est basé sur l'équation de conservation de la masse appliquée à la zone solide-liquide (zone pâteuse) et qui prend en compte à la fois une déformation subie par le squelette solide perpendiculairement aux dendrites et l'appel de liquide induit par le retrait de solidification. Le modèle introduit une chute de pression dans les films liquides au-delà de laquelle la cavitation, i.e. la germination d'un premier pore, a lieu. Des observations in situ sur des alliages organiques transparents permettent de mettre en évidence l'importance de la coalescence entre les dendrites; en effet, la prise de rigidité mécanique de la zone pâteuse se fait par l'établissement de ponts solides entre les dendrites. Enfin, le critère est appliqué successivement à la coulée semi-continue d'alliages d'aluminium, à la coulée continue des aciers et au traitement par laser des superalliages.
\end{abstract}

Mots clés : Solidification / fissuration à chaud / coalescence / alliages organiques / coulée continue / alliages d'aluminium / aciers / superalliages

\begin{abstract}
Hot tearing: from organic alloys to superalloys. The appearance of solidification cracks or hot tears during casting is a serious defect. EPF-Lausanne has developed a criterion for the appearance of hot tears which is based upon a mass balance performed over the liquid and solid phases and which takes into accounts for the tensile deformation of the solid skeleton perpendicular to the growing dendrites and for the induced interdendritic liquid feeding. This model introduces a critical pressure drop beyond which cavitation, i.e., nucleation of a first void, occurs. In situ observations on transparent organic alloys point our the importance of coalescence between dendrites; indeed the mechanical rigidity of the mushy zone is completed by the appearance of solid bridges between the dendrites. Eventually, the criterion is applied successively to the semi-continuous casting of aluminium alloys, to the continuous casting of steel and to the laser treatment of superalloys.
\end{abstract}

Key words: Solidification / hot cracking / hot tearing / coalescence / organic alloys / continuous casting / aluminium alloys / steel / superalloys

\section{Introduction}

La fissuration à chaud, ou crique à chaud, est un défaut qui peut apparaître au cours de la solidification de divers matériaux et qui se rencontre dans de nombreux procédés de mise en forme allant de la coulée semicontinue d'alliages d'aluminium au traitement par laser de superalliages. Après avoir rappelé les bases d'un critère de fissuration à chaud récemment développé à l'EPFLausanne, nous aborderons le phénomène de la coalescence entre les dendrites qui est important dans l'étude de la fissuration à chaud car il détermine à quel moment

\footnotetext{
${ }^{a}$ Auteur correspondant : jean-marie.drezet@epfl.ch
}

la structure pâteuse développe une rigidité mécanique. Enfin, le critère sera appliqué à différents procédés et à des matériaux des plus divers allant des alliages organiques aux superalliages.

\section{Fissuration à chaud}

Durant la solidification, un alliage métallique subit inévitablement des gradients thermiques qui vont induire, d'une part des mouvements de convection dans le liquide, d'autre part des contraintes dans le solide. La transition entre ces deux phases se fait graduellement au niveau d'une zone pâteuse, où les deux phénomènes seront 
Nomenclature

\begin{tabular}{|cccc|}
\hline$\Delta \mathrm{p}$ & chute de pression $(\mathrm{Pa})$ & $\mu$ & viscosité $($ Pa.s $)$ \\
$\rho$ & densité & $\mathrm{G}$ & gradient thermique $\left(\mathrm{K}_{\mathrm{m}} \mathrm{m}^{-1}\right)$ \\
$\mathrm{g}$ & accélération de pesanteur $\left(\mathrm{N} . \mathrm{kg}^{-1}\right)$ & $\mathrm{V}_{\mathrm{T}}$ & vitesse des isothermes $\left(\mathrm{m} . \mathrm{s}^{-1}\right)$ \\
$\beta$ & facteur de retrait de solidification $(-)$ & $\mathrm{K}$ & perméabilité $\left(\mathrm{m}^{2}\right)$ \\
$\mathrm{h}$ & hauteur de liquide $(\mathrm{m})$ & $\dot{\varepsilon}$ & vitesse de déformation $\left(\mathrm{s}^{-1}\right)$ \\
$\mathrm{A}, \mathrm{B}$ & intégrale $\left(\mathrm{K}\right.$ et $\left.\mathrm{K}^{2}\right)$ & $\mathrm{f}_{\mathrm{s}}$ & fraction volumique de solide $(-)$ \\
\hline
\end{tabular}

présents mais de manière plus complexe : la déformation du squelette solide dépendra fortement de son état de cohérence et la convection a lieu dans un milieu poreux.

Deux types importants de défaut sont liés à une mauvaise alimentation en liquide de la zone pâteuse : la microporosité et la fissuration à chaud. Comme mentionné par Campbell [1], le premier défaut est associé à une chute de pression induite par le retrait de solidification (le solide étant en général plus dense que le liquide) combinée à une ségrégation préférentielle de gaz dans le liquide (par exemple l'hydrogène pour les alliages d'aluminium). La fissuration à chaud, quant à elle, est liée à des contraintes qui apparaissent dans le réseau dendritique cohérent et qui déforment la partie non-cohérente. Cette dernière est constituée de dendrites (colonnaires ou équiaxes) dont les bras n'ont pas encore totalement coalescés. Sous l'effet de contraintes en traction ou en cisaillement, le film de liquide encore présent entre les dendrites offre peu de résistance et des fissures pourront potentiellement se former si le liquide n'arrive plus à compenser ces variations de volume.

Un modèle simple de formation de microporosité est dû à Niyama [2] : il consiste à calculer la chute de pression dans la zone pâteuse en régime stationnaire.

En revanche, les modèles de fissuration à chaud développés jusqu'alors sont essentiellement basés sur l'intervalle ou le chemin de solidification $[1,3-5]$ : plus l'intervalle de solidification est grand, plus la tendance à la formation de fissures à chaud est élevée. Clyne et Davis [6] ont reconnu le fait que la fissuration à chaud se développait dans une région où une ouverture du réseau dendritique ne pouvait plus être compensée par un apport de liquide, alors que Feurer a lui vu la similitude qu'il peut y avoir entre porosité et fissuration à chaud [7], mais son approche ne prend en compte que le retrait de solidification.

Le critère développé récemment au Laboratoire de métallurgie physique de l'EPFL [8] reprend l'approche de Niyama pour la formation de porosité mais en incluant les déformations uniaxiales du squelette solide. Le modèle est résumé sur la figure 1. En faisant l'hypothèse de dendrites colonnaires croissant en régime stationnaire avec une vitesse $V_{\mathrm{T}}$, dans un gradient thermique $\mathrm{G}$, le liquide doit compenser d'une part le retrait de solidification et d'autre part des déformations éventuelles propagées par la zone cohérente. La pression du liquide interdendritique, $\mathrm{p}(\mathrm{x})$, aura donc tendance à décroître avec une augmentation de la fraction de solide (donc de la pointe des dendrites

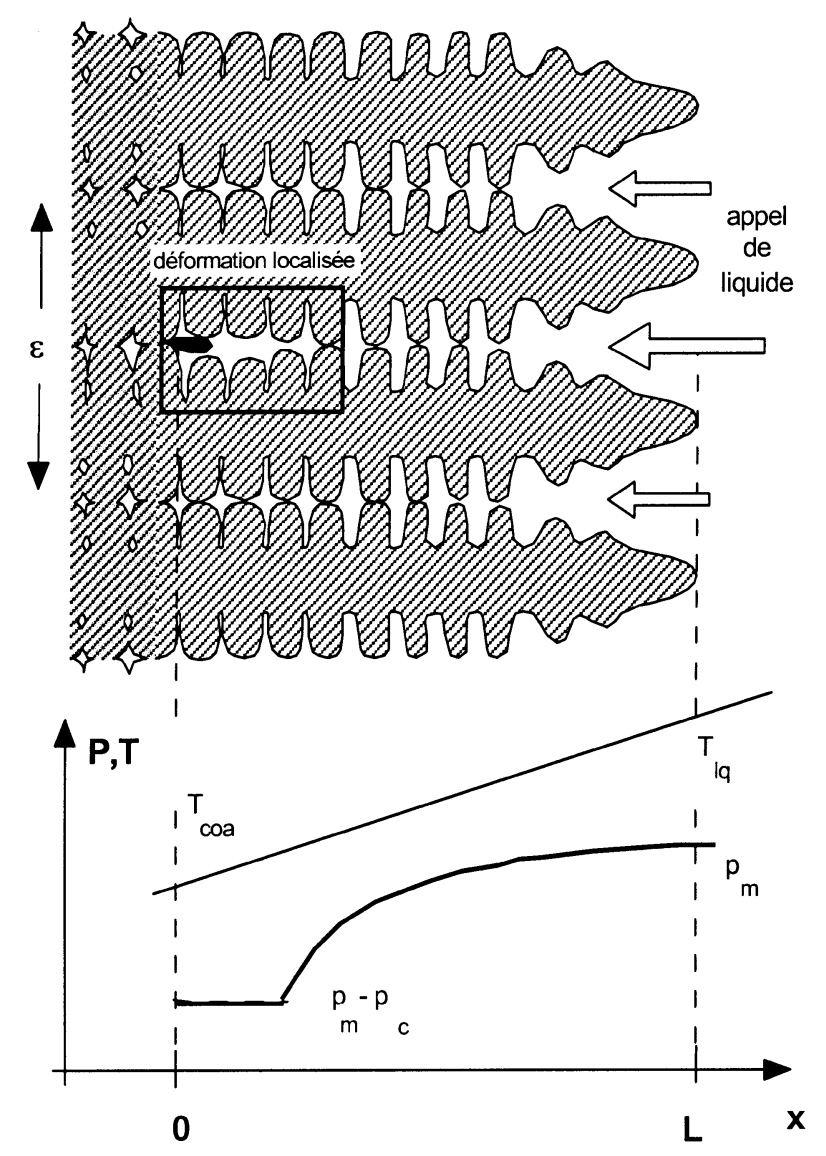

Fig. 1. Schéma de principe du modèle de fissuration à chaud.

vers l'eutectique). Si elle tombe en dessous d'une pression de cavitation, $\mathrm{p}_{\mathrm{c}}$, une fissure pourra être initiée (région noire de la Fig. 1). Le critère de fissuration sera donc donné par :

$$
\Delta \mathrm{p}_{\max }=\Delta \mathrm{p}_{\varepsilon}+\Delta \mathrm{p}_{\mathrm{sh}}-\rho \mathrm{gh}=\Delta \mathrm{p}_{\mathrm{c}}=\mathrm{p}_{\mathrm{o}}-\mathrm{p}_{\mathrm{c}}
$$

où $\Delta \mathrm{p}_{\varepsilon}$ et $\Delta \mathrm{p}_{\mathrm{sh}}$ sont les contributions à la dépression dues respectivement à la déformation et au retrait, $\rho g h$ étant la pression métallostatique à la pointe des dendrites. Ces dépressions sont calculées en appliquant l'équation de conservation de la masse sur un volume représentatif de la zone pâteuse. En supposant les densités du liquide et du solide constantes mais différentes, on introduit le facteur de retrait de solidification :

$$
\beta=\frac{\rho_{\mathrm{s}}}{\rho_{\mathrm{l}}}-1 \quad(\beta>0)
$$


De plus, la vitesse du liquide interdendritique est reliée à la pression via l'équation de Darcy $[2,8,9]$ :

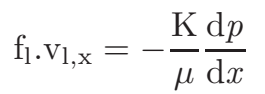

où $\mathrm{K}$ est la perméabilité de la zone pâteuse, $\mu$ la viscosité du liquide et $\mathrm{f}_{\mathrm{l}}=1-\mathrm{f}_{\mathrm{s}}$ la fraction volumique de liquide. $\mathrm{K}$ est donnée par la relation de Carman-Kozeny [8] :

$$
\mathrm{K}\left(\mathrm{f}_{\mathrm{s}}\right)=\frac{\lambda_{2}^{2}}{180} \frac{\left(1-\mathrm{f}_{\mathrm{s}}\right)^{3}}{\mathrm{f}_{\mathrm{s}}^{2}}
$$

où $\lambda_{2}$ est l'espacement des bras secondaires des dendrites. En combinant ces équations et en intégrant sur la longueur de la zone pâteuse avec un changement de variable $\mathrm{d} x=\mathrm{d} T \mathrm{G}^{-1}$, on obtient finalement les deux contributions à la chute de pression :

$$
\begin{aligned}
& \Delta \mathrm{p}_{\mathrm{sh}}+\Delta \mathrm{p}_{\mathrm{mec}}=\frac{180 \mu}{\mathrm{G} \lambda_{2}^{2}}\left[\mathrm{~V}_{\mathrm{T}} \beta \mathrm{A}+\frac{(1+\beta) \mathrm{B} \dot{\varepsilon}}{\mathrm{G}}\right] \\
& \operatorname{avec} \mathrm{A}=\int_{T_{\mathrm{coa}}}^{T \operatorname{liq}} \frac{\mathrm{f}_{\mathrm{s}}^{2} \mathrm{~d} T}{\left(1-\mathrm{f}_{\mathrm{s}}\right)^{2}} \quad \text { et } \mathrm{B}=\int_{T_{\mathrm{coa}}}^{T_{\text {liq }}} \frac{\mathrm{f}_{\mathrm{s}}^{2} \cdot \int_{T_{\mathrm{coa}}}^{T} \mathrm{f}_{\mathrm{s}} \mathrm{d} T}{\left(1-\mathrm{f}_{\mathrm{s}}\right)^{3}} \mathrm{~d} T
\end{aligned}
$$

Les intégrations se font de la température de coalescence des dendrites, $T_{\text {coa }}$, à la température du liquidus de l'alliage. Il est à noter que lorsque $T_{\text {coa }}$ tend vers $T_{\text {sol }}$ (température de fin de solidification), les intégrales A et B tendent vers l'infini car la perméabilité du milieu tend vers 0 . Le chemin de solidification, $\mathrm{f}_{\mathrm{s}}(T)$, peut être donné par un modèle de solidification tel que celui de Clyne et Kurz prenant en compte la rétrodiffusion de soluté dans le solide [10] ou des modèles numériques.

Le critère de fissuration à chaud donné par l'équation (1) requiert deux paramètres : la pression de cavitation $\mathrm{p}_{\mathrm{c}}$ à laquelle la fissure germe dans le film interdendritique de liquide et la fraction de solide, $\mathrm{f}_{\text {coa }}$, ou la température critique, $T_{\text {coa }}$, à laquelle le réseau dendritique devient cohérent (fraction de solide ou température de coalescence des bras). Dans la section suivante, la mesure de cette température est présentée dans le cas d'alliages organiques transparents.

\section{Observation sur les alliages organiques}

Des observations sur les organiques ont été conduites à l'aide du dispositif expérimental décrit sur la figure 2 [11,12]. À l'aide d'un moteur électrique, les dendrites sont écartées dans le sens perpendiculaire au gradient thermique. L'apparition de fissures à chaud est alors filmée par une caméra située au-dessus de la cellule contenant l'organique.

Le binaire succinonitrile-0.5 \% pds acétone, SCNacétone, est un organique transparent; sa température

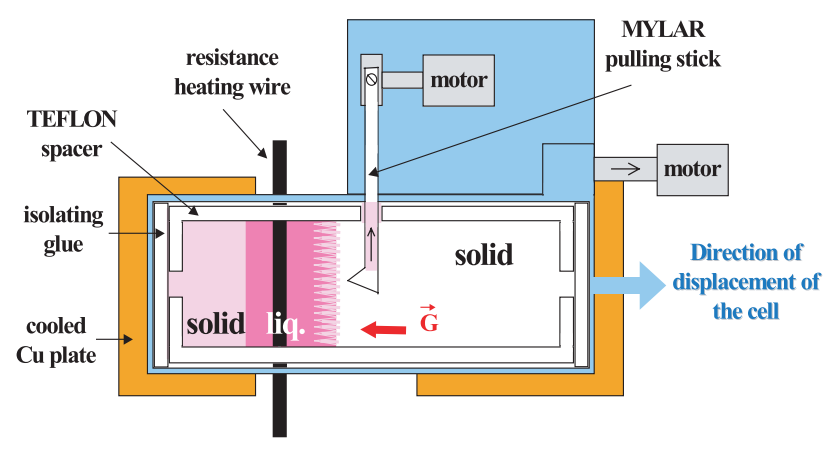

Fig. 2. Dispositif utilisé pour l'observation in situ de fissures à chaud sur des organiques.

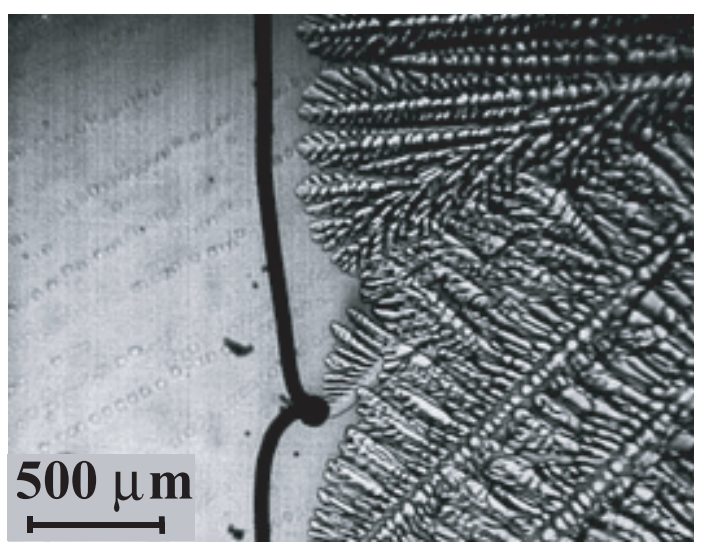

Fig. 3. Mesure de la température du liquidus à l'aide du thermocouple inséré dans la cellule.

de fusion se situe vers $55^{\circ} \mathrm{C}$. Aux faibles fractions de solide, l'ouverture du réseau interdendritique crée un appel de liquide enrichi en soluté et aucune fissure n'apparaît (on parle alors de crique guérie). En revanche, aux fractions plus élevées, des fissures s'initient sur des défauts tels que des micropores, en raison de la faible perméabilité du réseau dendritique.

En insérant un thermocouple dans la cellule et en observant le front de solidification, il est possible de déterminer la température du liquidus de l'alliage (et donc sa concentration) au passage des pointes de dendrites (cf. Fig. 3) et les températures de coalescence intergranulaire, Tcg (cf. Fig. 4), et de coalescence intragranulaire, Tcd (cf. Fig. 5). Dans les conditions expérimentales utilisées, le liquidus se situe autour de $54.8^{\circ} \mathrm{C}$, et Tcd et Tcg autour de $43.8^{\circ} \mathrm{C}$ et $27.6^{\circ} \mathrm{C}$, respectivement.

En utilisant le modèle de micro-ségrégation de Clyne-Kurz [13], on peut remonter aux fractions de solide correspondant à Tcg et Tcd. La figure 6 montre les valeurs des intégrales A et B en fonction de la fraction de solide à la coalescence. La coalescence à l'intérieure d'un même grain se fait aux environs de $95 \%$ de solide alors qu'elle a lieu à des fractions de solide plus élevées, $99 \%$, entre deux grains d'orientation différente. Évidemment, cette valeur dépend fortement de la désorientation des grains et de la nature chimique du film liquide (présence d'éléments fortement ségrégés). Les intégrales A et B 


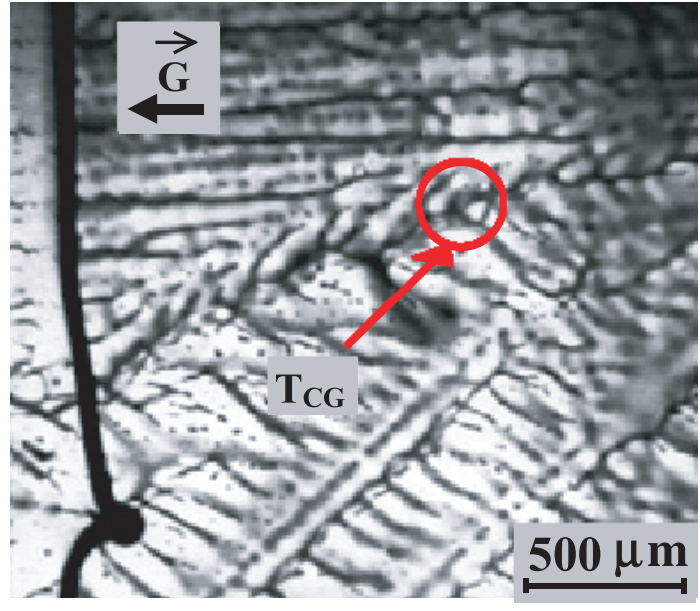

Fig. 4. Mesure de la température de coalescence des grains, Tcg.

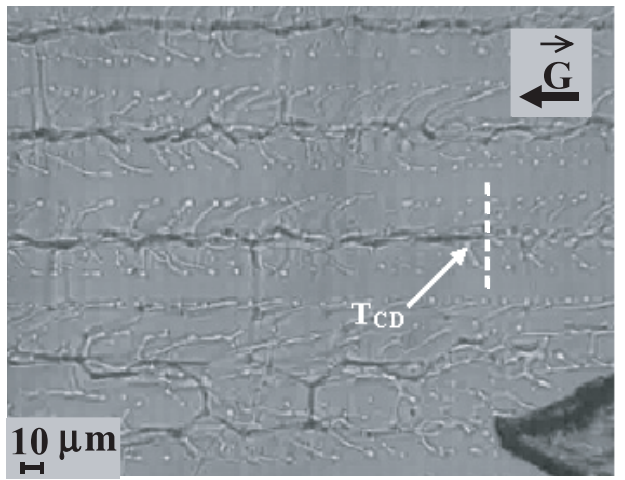

Fig. 5. Mesure de la température de coalescence des dendrites d'un même grain, Tcd.

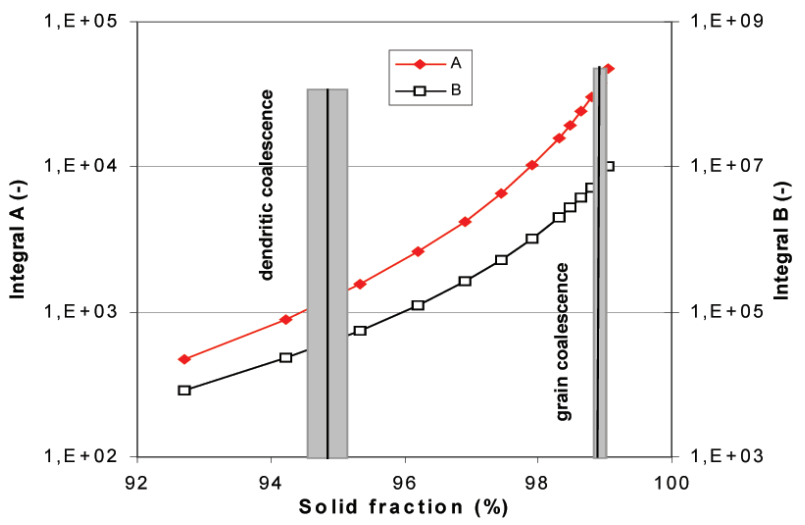

Fig. 6. Intégrales A et B (normalisées) en fonction de la fraction de solide à la coalescence.

sont environ deux ordres de grandeur plus élevées dans le cas intergranulaire : ceci signifie que pour des conditions thermomécaniques identiques, la chute de pression (cf. Éqs. (1) et (5)) sera beaucoup plus forte dans les films liquides intergranulaires. Autrement dit, l'apparition de criques se fait préférentiellement entre les grains et non à l'intérieur de grains.

\section{Coalescence}

Un paramètre fondamental du critère de fissuration à chaud décrit plus haut est la température ou la fraction solide de coalescence, inter-granulaire pour une structure équiaxe, intra-granulaire pour une structure monocristalline. En effet, les intégrales A et B présentées ci-dessus dépendent très fortement de la température de coalescence. Il est donc primordial de déterminer à quelle température la zone pâteuse peut supporter des contraintes en traction et en cisaillement. Cette prise de rigidité mécanique est liée à l'établissement de ponts solides entre les dendrites, i.e. au phénomène de coalescence. La figure 7 présente une vue schématique des atomes lorsque deux grains séparés par un film liquide se rapprochent. L'énergie libre pour un matériau pur est aussi représentée schématiquement. Lorsque les deux grains sont suffisamment éloignés, l'énergie libre vaut alors $2 \gamma_{\mathrm{sl}}$ puisque deux interfaces solide-liquide sont présentes. Cette énergie devient $\gamma_{\mathrm{gb}}$ lorsque le film liquide disparaît. Il est à noter que cette énergie est nulle si les grains ont une même orientation, par exemple si les dendrites appartiennent au même grain.

La coalescence entre deux grains solides dépend donc, entre autre, de la différence $2 \gamma_{\mathrm{sl}}-\gamma_{\mathrm{gb}}$. Sur la figure 8 , l'énergie de surface des deux interfaces solide-liquide est représentée en fonction de l'épaisseur du film liquide, $h$. Si $\gamma_{\mathrm{gb}}$ est plus petit que $2 \gamma_{\mathrm{sl}}$ (cas a) les deux interfaces ont tendance à s'attirer; la situation est dite attractive. C'est le cas pour deux grains de désorientation nulle ou deux dendrites d'un même grain puisque dans ce cas $\gamma_{\mathrm{gb}}$ est nul. Si par contre $\gamma_{\mathrm{gb}}$ est plus grand que $2 \gamma_{\mathrm{sl}}$ (cas b) les deux interfaces ont tendance à se repousser (situation répulsive, cas b). Dans la majeure partie des métaux, l'énergie d'interface entre deux grains différents, $\gamma_{\mathrm{gb}}$, est plus grande que $2 \gamma_{\mathrm{sl}}$. Cela conduit à une situation répulsive et à la présence de film liquide même en dessous de la température de fusion, $T_{\mathrm{m}}$. Cette situation est illustrée sur la figure 8c. À une surfusion donnée, $\Delta T>0$, l'énergie libre de Gibbs du liquide par unité de surface est une fonction linéaire de l'épaisseur du film liquide, $\mathrm{h}$; elle est donnée par $\left(\mathrm{G}_{\mathrm{l}}-\mathrm{G}_{\mathrm{s}}\right) \mathrm{h}=\left(\mathrm{L} \Delta T / T_{\mathrm{m}}\right) \mathrm{h}>0$, où $\mathrm{L}$ est la chaleur latente de fusion par unité de volume. En supposant que la variation d'énergie de surface est donnée par (cf. Fig. 8) :

$$
\gamma(\mathrm{h})=2 \gamma_{\mathrm{sl}}+\left(\gamma_{\mathrm{gb}}-2 \gamma_{\mathrm{sl}}\right) \exp \left(\frac{-\mathrm{h}}{\delta}\right)
$$

on peut montrer qu'un minimum d'énergie existe pour $\mathrm{h}=\mathrm{h}^{*}$. Un film liquide disparaîtra alors dans une substance pure à une surfusion de coalescence, $\Delta T_{\mathrm{b}}$, donnée par :

$$
\Delta T_{\mathrm{b}}=\Delta T_{\mathrm{h}^{*}=0}=\frac{\left(\gamma_{\mathrm{gb}}-2 \gamma_{\mathrm{sl}}\right) T_{\mathrm{m}}}{\mathrm{L}} \frac{1}{\delta}
$$

où $\delta$ est une distance caractéristique sur laquelle les atomes « ressentent » la présence d'une interface (joint de grain ou interface solide-liquide). $\delta$ est de l'ordre de quelques nanomètres dans les métaux. Le cas $\gamma_{\mathrm{gb}}=2 \gamma_{\mathrm{sl}}$ correspond à une interface «neutre » puisque $\Delta T_{\mathrm{h}^{*}=0}=0$, 

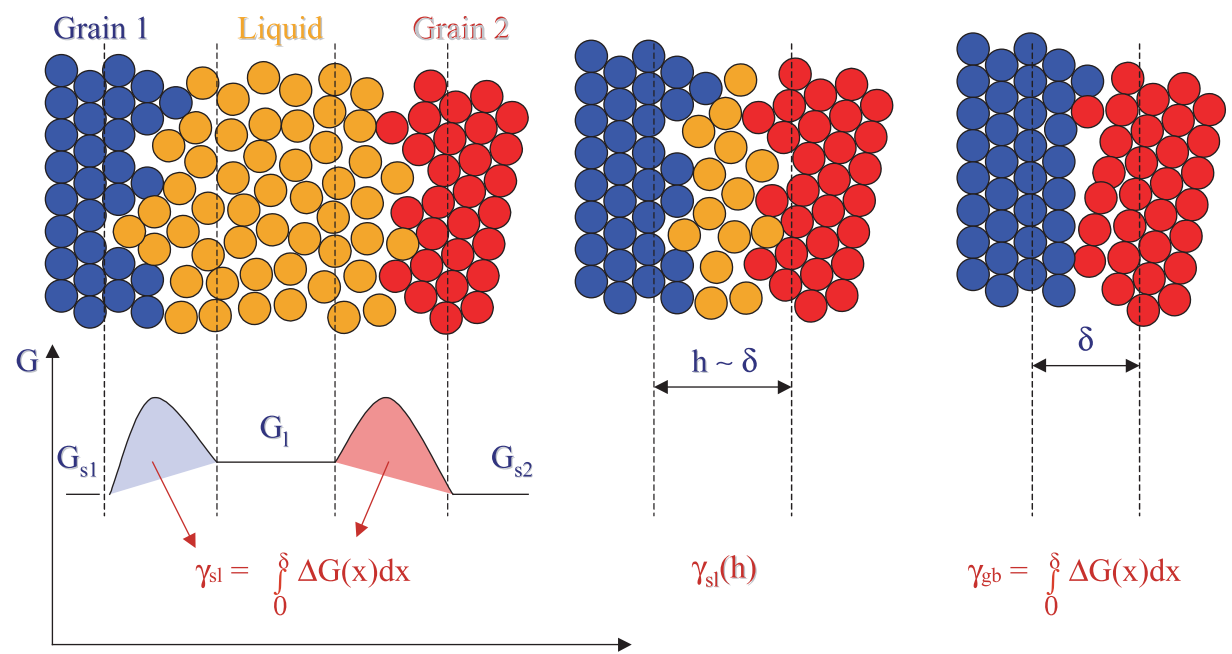

Fig. 7. Représentation atomistique de la disparition d'un film liquide entre deux grains solides [14].

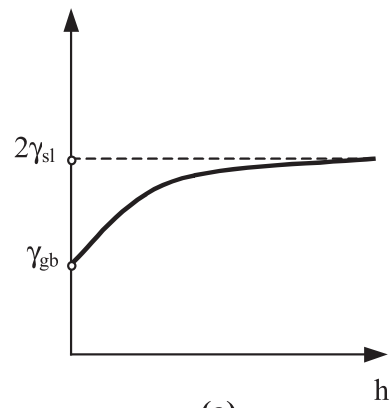

(a)

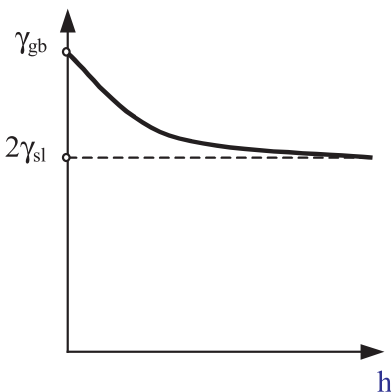

(b)

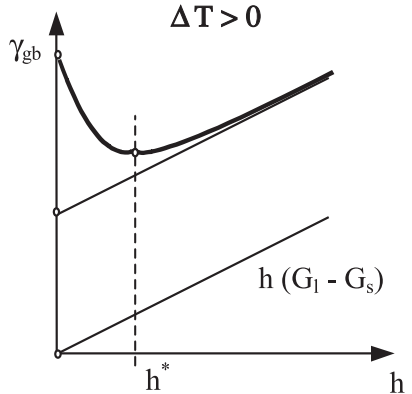

(c)

Fig. 8. Énergie de surface lorsque deux grains solides sont rapprochés [14].

i.e., un tel joint de grain se solidifie à $T_{\mathrm{m}}$ pour une substance pure.

Dans les alliages, la situation est plus complexe en raison de la diffusion des éléments d'alliage. Dans leur étude sur la coalescence, Rappaz et al. [14] ont utilisé des méthodes de suivi de front et de champ de phase pour étudier l'interaction entre la courbure de l'interface solide-liquide et la diffusion de solutés dans les phases solide et liquide. Ces travaux permettront de mieux déterminer la température de coalescence et sa dépendance avec le type de microstructure étudié, équiaxe, globulaire ou colonnaire.

\section{Application à la coulée semi-continue d'alliages d'aluminium}

Le critère de fissuration à chaud décrit plus haut a été appliqué aux alliages d'aluminium coulés sous forme de billettes ou de lingots [15]. Le procédé dit de coulée semi-continue, est utilisé industriellement pour obtenir les semi-produits qui seront ensuite extrudés (billettes) ou laminés (lingots). Cependant, l'apparition de criques au centre du lingot ou de la billette (cf. Fig. 9) nécessite l'utilisation de sondes ultra-sonores et abaisse particulièrement la productivité, notamment pour les alliages très sensibles, en limitant la vitesse de coulée.

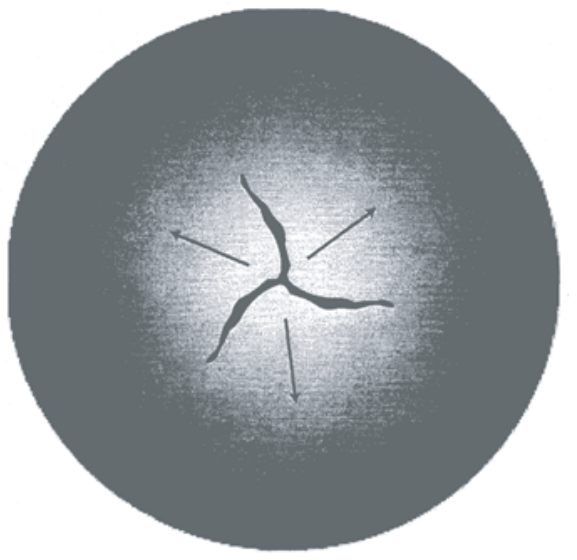

Fig. 9. Fissure au centre d'une billette [16].

La figure 10 présente la chute de pression calculée par ABAQUS dans la zone pâteuse (en $\mathrm{kPa}$ ) pour une billette de diamètre $200 \mathrm{~mm}$ coulée à $120 \mathrm{~mm} \cdot \mathrm{min}^{-1}$.

Il apparaît clairement que le centre de la billette (zone rouge) présente les plus fortes chutes de pression bien que la pression métallostatique, qui a un effet positif (cf. Éq. (1)) y soit très élevée. La forte sensibilité à la fissuration à chaud présente au centre de la billette s'explique par les vitesses de déformation élevées en fond de puits 


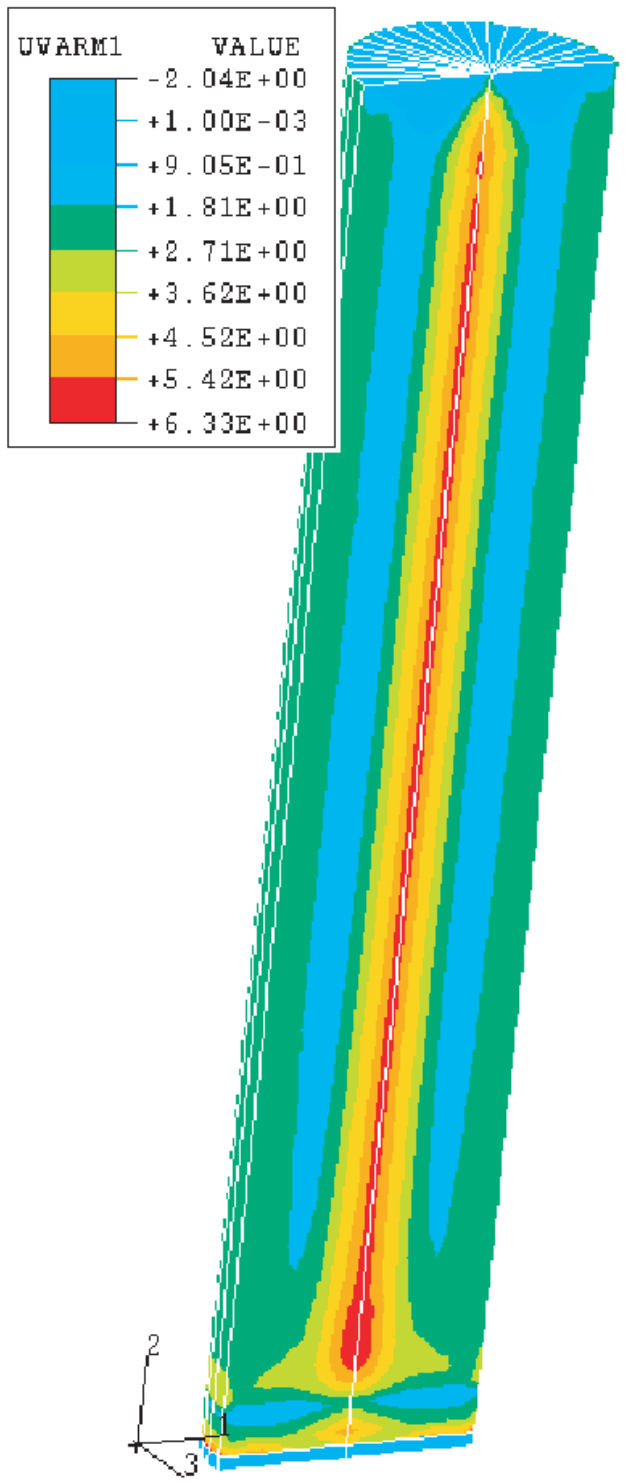

Fig. 10. Chute de pression dans la zone pâteuse (en $\mathrm{kPa}$ ) pour une billette de diamètre $200 \mathrm{~mm}$ coulée à $120 \mathrm{~mm} \cdot \mathrm{min}^{-1}$.

liquide lors de la coulée. On peut noter que la surface de la billette présente aussi une légère sensibilité à la fissuration à chaud. La figure 11 représente la chute de pression calculée le long de l'axe de symétrie de la billette : cette quantité passe par un maximum au démarrage de coulée puis redescend quelque peu vers une valeur stationnaire d'environ $5.5 \mathrm{kPa}$. Si la dépression de cavitation est plus élevée que $5.5 \mathrm{kPa}$, alors une fissure initiée au démarrage de coulée se propagera tout le long du lingot alors que dans le cas contraire, elle se limitera au pied de coulée.

\section{Application à la coulée continue des aciers}

Le principe de la coulée continue des aciers est décrit à la figure 12 [17]. Des fissures intercolonnaires sont aussi souvent observées (cf. Fig. 13) : elles sont liées aux

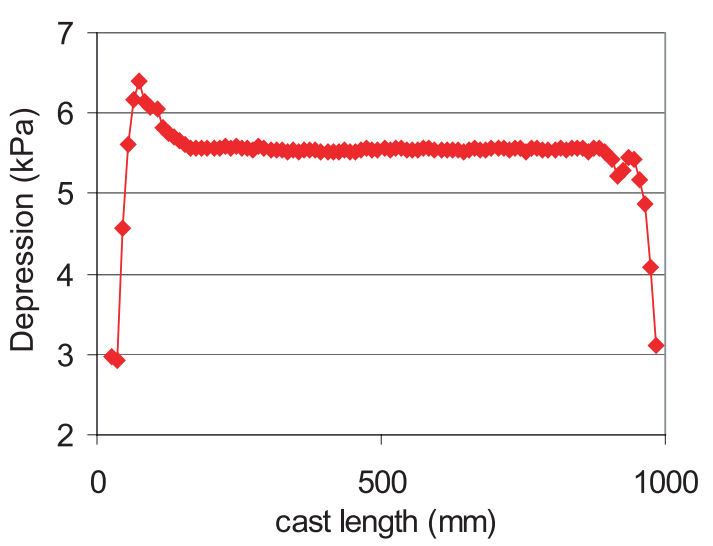

Fig. 11. Chute de pression le long de l'axe de la billette.

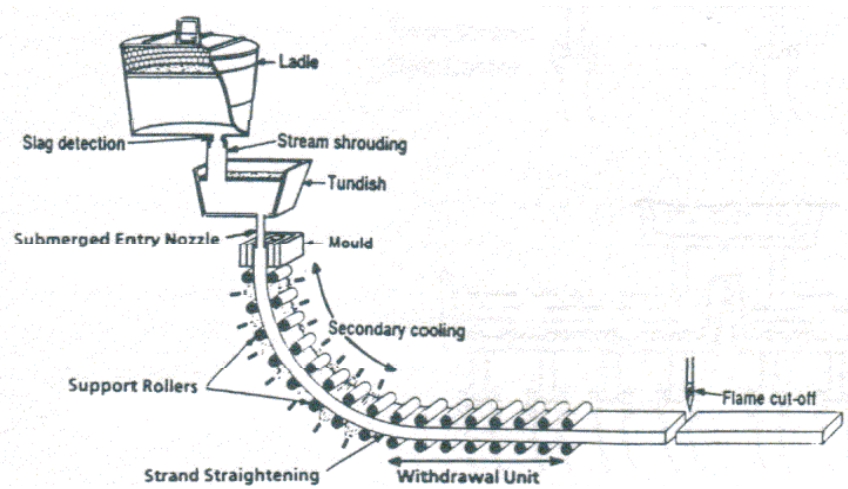

Fig. 12. Principe de la coulée continue des aciers.

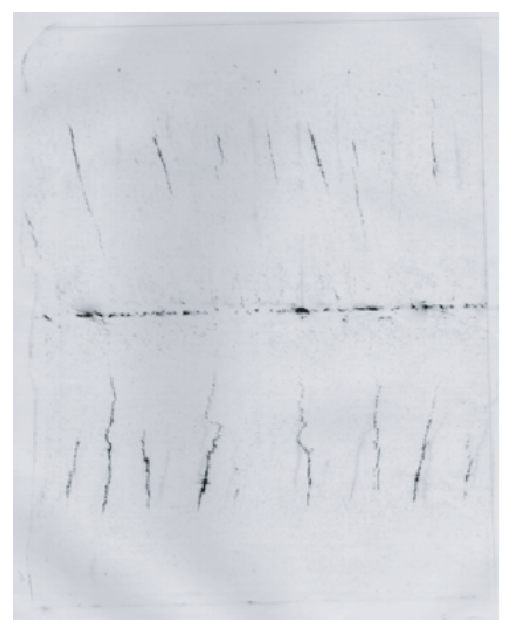

Fig. 13. Fissures révélées par une attaque au soufre.

déformations mécaniques que subit la croûte du lingot telles que le gonflement entre les rouleaux dû à la pression métallostatique, ou le redressement de la brame en position horizontale. Les éléments qui ségrègent fortement, tels que $\mathrm{S}$ ou $\mathrm{P}$, sont connus pour rendre les aciers très sensibles à la fissuration à chaud : leur teneur est très contrôlée par les sidérurgistes.

L'approche de la fissuration à chaud présentée plus haut, a été étendue au cas des aciers [18]. Pour ceuxci, il est primordial de prendre en compte le fait que 


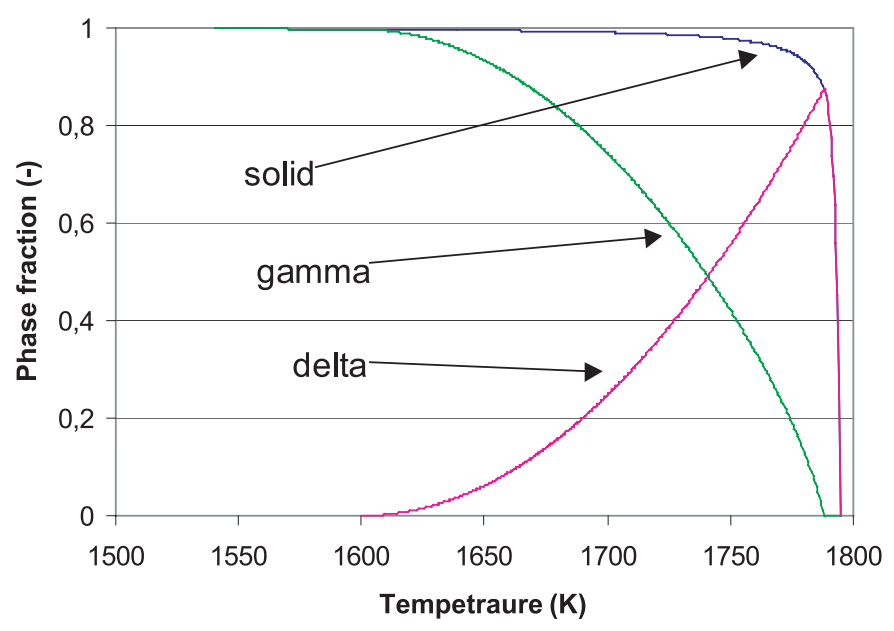

Fig. 14. Distribution des phases au cours de la solidification de l'acier Fe-0.15C-0.03P.

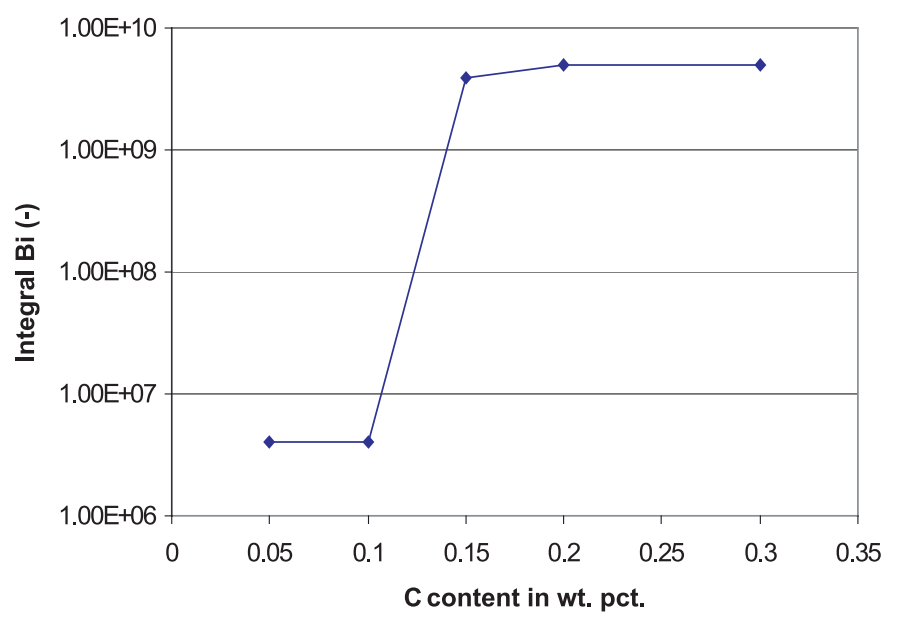

Fig. 15. Intégrale $\mathrm{B} \gamma$ en fonction de la teneur en C.

deux phases, la ferrite $\delta$ et l'austénite $\gamma$, peuvent se former lors de la solidification à cause de la présence d'une réaction péritectique. Le modèle de Clyne-Wolf et Kurz [19] permet de calculer la distribution des phases présentes lors de la solidification d'un alliage ternaire FeC-P. Le résultat est présenté pour l'acier Fe-0.15C-0.03P (\% pds) sur la figure 14. Ce type de courbes est nécessaire pour calculer les intégrales A et B des phase $\delta$ et $\gamma$. La figure 15 montre l'influence de la teneur en $\mathrm{C}$ sur l'intégrale $\mathrm{B} \gamma$, autrement dit sur la sensibilité à chaud de l'acier. Pour des teneurs en $\mathrm{C}$ inférieures à $0.1 \%$, l'acier se solidifie uniquement en phase $\delta$ alors que pour des teneurs plus élevées, la solidification se termine par la phase $\gamma$. La courbe présentée sur la figure 15 est tout à fait en accord avec les mesures de l'indice de fissuration subsurfacique [19], reproduites à la figure 16 .

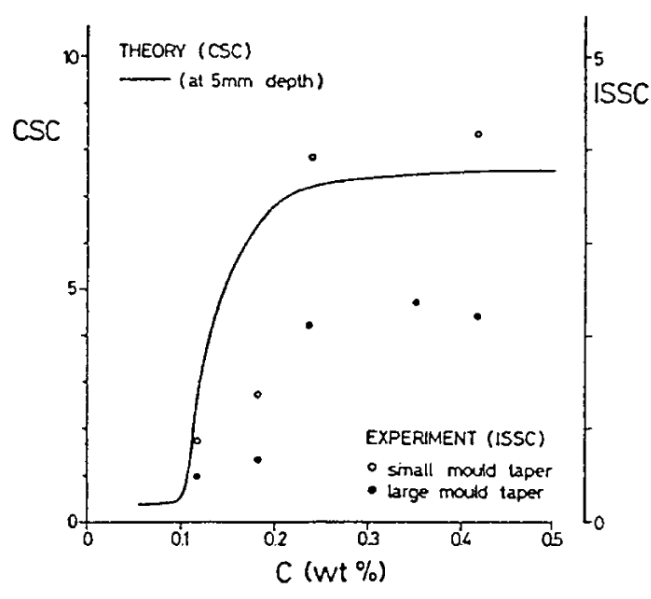

Fig. 16. Indice de fissuration sub-surfacique en fonction de la teneur en C [19].

\section{Application au traitement par laser des superalliages}

Les procédés de traitement laser comme celui présenté en figure 17, ont un potentiel d'application pour l'augmentation de la durée de vie de ces pièces monocristallines. Un traitement monocristallin peut être obtenu lors de rechargement et de plaquage par laser, principalement en raison des gradients thermiques élevés relatifs aux procédés [20-22]. Ainsi, la réparation monocristalline d'aubes défectueuses et le dépôt de couche de protection monocristalline sont envisageables. Ce dernier cas permettrait d'augmenter la résistance en fatigue thermique des aubes par une augmentation de la compatibilité mécanique du dépôt avec le substrat monocristallin.

Le succès d'un traitement laser monocristallin nécessite, entre autres, le contrôle de plusieurs étapes de la solidification. Il s'agit d'abord de garantir une initiation de la solidification par croissance épitaxiale avec le substrat (i.e. sans germination) afin de reproduire l'orientation cristallographique du substrat dans le dépôt. De plus, il faut assurer une croissance colonnaire, afin de maintenir l'orientation cristallographique dans la totalité du dépôt et d'éviter l'initiation de fissures en fin de solidification par mécanisme de fissuration à chaud. Les criques à chaud sont identifiables par l'aspect interdendritique du faciès de rupture, comme illustré en figure 18. Cette observation au MEB a été faite sur un bain liquide final de traitement de refusion par laser de superalliages monocristallins CMSX-4. Une croissance colonnaire de dendrites, perpendiculaire à la photographie, est observée en raison du retrait du liquide final (retassure). À la base des troncs dendritiques, une fissure interdendritique est présente.

Les superalliages à base de nickel sont connus pour être difficilement soudables en raison de leur forte susceptibilité à la fissuration à chaud. Cependant, cette susceptibilité est fortement influencée par les teneurs en éléments d'alliage. Un intervalle de solidification plus faible ou une fraction volumique d'eutectique plus importante diminue la zone critique pour laquelle un film liquide continu est 


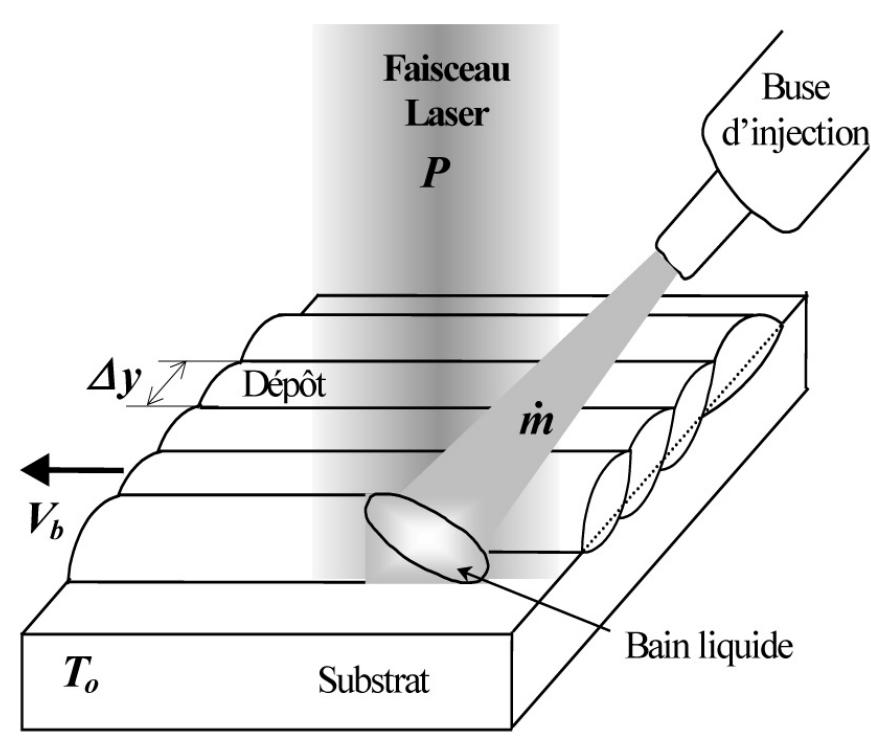

Fig. 17. Représentation schématique du procédé de recouvrement par projection de poudre métallique dans un bain liquide contrôlé par source laser [22].

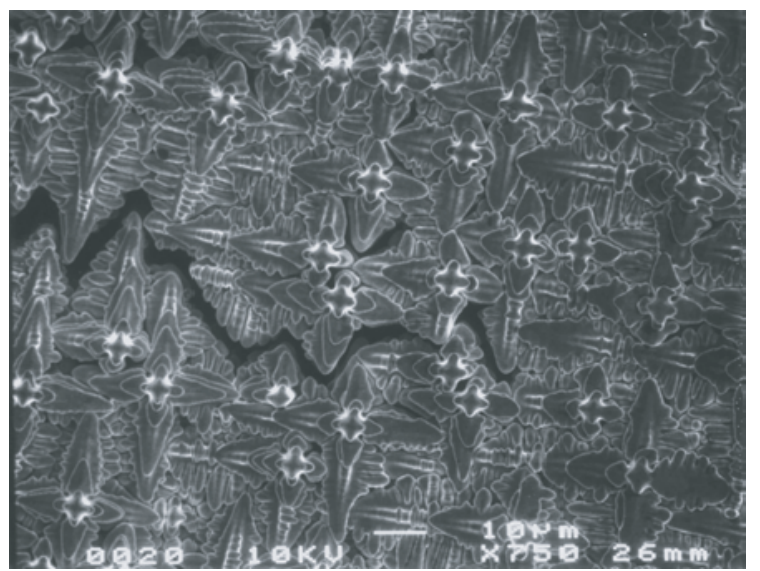

Fig. 18. Observation au MEB d'une fissure interdendritique.

présent entre les dendrites. La figure 19 montre deux sections de dépôts pour deux alliages NiCrAlY de différentes compositions, A1 et A2.

Un grand nombre de fissures internes est observé lors de la déposition de l'alliage A1 (image du haut) alors qu'un dépôt sans fissures est obtenu avec l'alliage A2 (image du bas).

L'influence des éléments d'alliages sur la susceptibilité à la fissuration peut être mise en évidence par le biais de la comparaison des intégrales A et B de chaque composition. En supposant que les paramètres $\beta, \mu$ et $\Delta \mathrm{P}_{\mathrm{c}}$ sont identiques pour des alliages de composition proche, la susceptibilité à la fissuration à chaud de chaque alliage sera, pour des conditions de solidification $\mathrm{G}$ et $\mathrm{V}_{\mathrm{T}}$ identiques, proportionnelle aux paramètres $\mathrm{A}$ et $\mathrm{B}$. Ceux-ci peuvent être évalués avec l'utilisation de modèles de microségrégation ou sur la base de mesures expérimentales de la fraction solide $\mathrm{f}_{\mathrm{s}}(T)$ par analyse thermique différentielle (DTA). Bien que cette analyse reste approximative et présente un

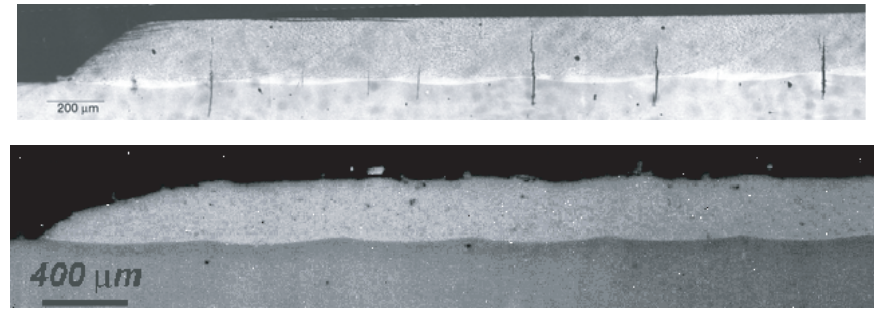

Fig. 19. Sections transverses de dépôts monocristallins de 2 alliages NiCrAlY sur CMSX-4 (6 traces laser successives).

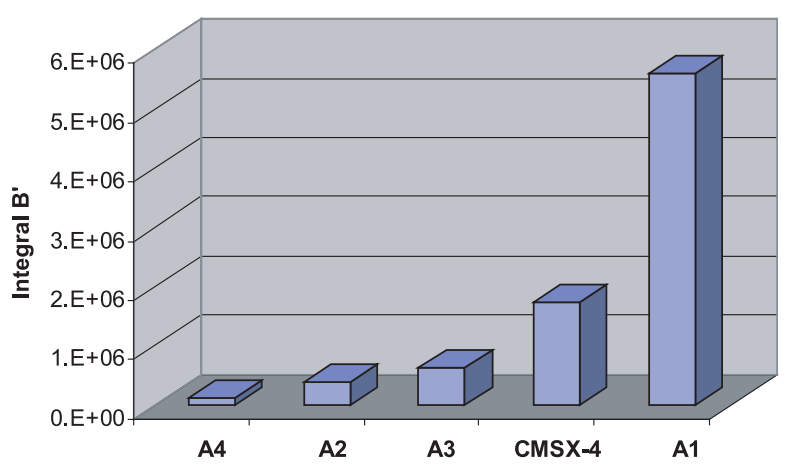

Fig. 20. Comparaison de la susceptibilité à la fissuration à chaud de différents alliages.

certain nombre d'incertitudes, elle permet toutefois une comparaison qualitative de différents alliages.

La figure 20 montre le classement par susceptibilité croissante à la fissuration à chaud de différents alliages $\mathrm{NiCrAlY}$ : il apparaît clairement que l'alliage A1 est beaucoup plus sensible que l'alliage A2, comme montré sur la figure 19. L'évaluation des intégrales A et B pour un alliage donné s'avère alors être un outil indispensable pour définir de nouveaux matériaux de revêtement moins sensibles à la fissuration à chaud.

\section{Conclusion}

Le modèle de fissuration à chaud présenté ici et appliqué à différents procédés et matériaux semble prometteur : l'étude de la coalescence entre les grains au cours de la solidification permettra de mieux comprendre encore la prise de rigidité mécanique de la structure.

\section{Références}

[1] J. Campbell, Castings, Butterworth-Heinemann, Oxford, 1991

[2] E. Niyama, T. Uchida, M. Morikawa, S. Saito, AFS Int. Cast Metals J. (1982) 52-63

[3] R. Jauch, Stahl u. Eisen 98 (1978) 244-254

[4] Y.F. Guven, J.D. Hunt, Cast Metals 1 (1988) 104-111

[5] D. Warrington, D.G. McCartney, Cast Metals 2 (1989) 134-143

[6] T.W. Clyne, G.J. Davies, Brit. Found. 74 (1981) 65-73. See also : Brit. Found. 68 (1975) 238-244 
[7] U. Feurer, Giesserei Forsch. 2 (1976) 75-80

[8] M. Rappaz, J.-M. Drezet, M. Gremaud, Met. Mater. Trans. vol. 30A (1999) 449-455

[9] J. Ampuero, Ch. Charbon, A.F.A. Hoadley, M. Rappaz, in Materials Processing in the Computer Age, V.R. Voller, M.S. Stachowicz, B.G. Thomas (ed.), TMS Publ., Warrendale, Pennsylvania, 1991, pp. 377-388

[10] W. Kurz, D.J. Fisher, Fundamentals of Solidification, Trans. Tech. Publication, third edition, Aedermannsdorf, 1989

[11] I. Farup, J.-M. Drezet, M. Rappaz, Acta Materialia 49 (2001) 1261-1269

[12] P.-D. Grasso, J.-M. Drezet, I. Farup, M. Rappaz, Direct observation of hot tear deformation in organic alloys, presented at the 7th Conf. on Advanced Materials and Processes, EuroMat 01, Rimini, June 2001

[13] T.W. Clyne, W. Kurz, Metall. Trans. A 12A (1981) 965

[14] M. Rappaz, A. Jacot, W.J. Boettinger, Last stage solidification of alloys, A theoretical study of dendrite arm and grain coalescence, Met. Mater. Trans. 34A (2003) 467-479

[15] J.-M. Drezet, M. Rappaz, Prediction of hot tears in DC cast billets, in Light Metals, J.-L. Anjier (ed.), TMS, New Orleans, 2001, pp. 887-893

[16] I. Farup, Thermally induced deformations and hot tearing during direct chill casting of aluminium, $\mathrm{Ph}$.D. work, University of Oslo, Norway, 2000
[17] Continuous Casting of Steel, W.R. Irving, The Institute of Materials, 1993, London

[18] J.-M. Drezet, M. Gremaud, R. Graf, M. Gaümann, A new hot tearing criterion for steel, Proceedings of the 4th European Continuous Casting Conference, IOM communications, Birmingham, UK, October 2002, pp. $755-763$

[19] T.W. Clyne, M. Wolf, W. Kurz, The effect of melt composition on solidification cracking of steel, with particular reference to continuous casting, Met. Trans. 13B (1982) 259

[20] M. Gäumann, P.-H. Jouneau, J.-D. Wagnière, W. Kurz, Epitaxial laser metal forming on a single crystal superalloy, in M. Geiger, F. Vollersten (ed.), Proc. of the LANE'97, Sep. 23-26, 1997, Erlangen, Germany, vol. 2, 651

[21] M. Gäumann, C. Bezençon, P. Canalis, W. Kurz, Single-crystal laser deposition of superalloys : processing-microstructure maps, Acta Mater. 49 (2001) 1051-1062

[22] C. Bezençon, S. Mokadem, J.-D. Wagnière, J.-M. Drezet, W. Kurz, Solidification monocristalline et fissuration à chaud lors de traitement laser de superalliages base nickel presenté au Séminaire Européen Recherche/Industrie sur les applications des lasers de Puissance (Laser'ap 01), Sarlat, France, 2001, pp. 81-100 